\title{
TROP2 promotes cell proliferation and migration in osteosarcoma through PI3K/AKT signaling
}

\author{
QING-ZHI GU, ABULIMITI NIJIATI, XING GAO, KAI-LIANG TAO, \\ CHENG-DUO LI, XUE-PENG FAN and ZHENG TIAN
}

Department of Bone Tumor, The First Affiliated Hospital of Xinjiang Medical University, Urumqi, Xinjiang 830054, P.R. China

Received January 8, 2018; Accepted May 15, 2018

DOI: $10.3892 / \mathrm{mmr} .2018 .9083$

\begin{abstract}
Human trophoblast cell surface antigen 2 (TROP2) has been noted to serve an important role in the proliferation and migration of various types of human cancers. However, the potential role and the molecular mechanisms of TROP2 in osteosarcoma (OS) remain largely unclear. In the present study, high expression of TROP2 in human OS tissues and cell lines was observed. Overexpression of TROP2 promoted the proliferation and migration of OS cell lines, while TROP2 knockdown markedly decreased cell growth and migration. Furthermore, it was revealed that TROP2 overexpression significantly activated the phosphoinositide 3-kinase/protein kinase B (PI3K/AKT) signaling pathway. Collectively, these results suggested that TROP2 may promote OS cell proliferation and migration via PI3K/AKT signaling and may serve as a novel treatment target for OS.
\end{abstract}

\section{Introduction}

Osteosarcoma (OS) is the most common malignant tumor of the skeletal system, with high rates of local invasion and early metastasis. OS mostly occurs among children and adolescents, with a reported incidence rate of 4-5 per million (1). The overall survival rate of patients with OS is reported to be $60-80 \%$ (2-4). However, despite the advances in multiagent chemotherapy and surgical technique, the survival rate of patients with locally advanced or metastatic tumors at diagnosis is still very low $(<20 \%)(5-9)$. Thus, there is urgent need to reveal detailed signal pathways involved in OS pathogenesis and the molecular mechanism of its metastasis, which may provide novel therapeutic targets for the clinical management of OS.

Correspondence to: Dr Zheng Tian, Department of Bone Tumor, The First Affiliated Hospital of Xinjiang Medical University, 393 Xinyi Road, Xinshi, Urumqi, Xinjiang 830054, P.R. China E-mail: zheng_tian_cn@outlook.com

Key words: trophoblast cell surface antigen 2, osteosarcoma, phosphoinositide 3-kinase/protein kinase B
Human trophoblast cell surface antigen 2 (TROP2) is a 36-kDa single-pass transmembrane protein encoded by the tumor-associated calcium signal transducer 2 (Tacstd2) gene, with low to no expression in normal tissues (10-13). Accumulating studies have demonstrated TROP2 to be a candidate tumor prognostic marker, which was highly expressed by various tumors, such as pancreatic cancer $(14,15)$, gastric cancer $(11,16)$, lung cancer $(17,18)$, ovarian cancer $(19)$, and colorectal cancers (20). TROP2 overexpression correlates with increased tumor recurrence, invasiveness, and poor clinical outcome (21-23). Although TROP2 has mostly been reported to be highly expressed in epithelial cancers, previous studies have also found TROP2 expression in stem cells in various tissue types, such as human and mouse prostate $(10,24)$. In addition, TROP2 is also expressed in bone tissues and regulates the proliferation and differentiation of bone marrow stromal cells (25-27). However, so far the potential role and the molecular mechanisms of TROP2 in OS remain largely unclear.

The present study aimed to investigate the role of TROP2 in OS. We evaluated the expression of TROP2 in human OS tissues and cell lines. Furthermore, the specific effects of TROP 2 on OS cells proliferation, cell migration, together with the possible mechanism involved in this process were also explored for the first time. The results of the present study suggest that TROP2 may be a potential prognostic biomarker and a potential therapeutic target for OS.

\section{Materials and methods}

Clinical tissue samples. This study was approved by the Medical Ethics Committee of The First Affiliated Hospital of Xinjiang Medical University (Urumqi, China). Ten OS specimens and paired adjacent normal bone tissues were collected during surgery from OS patients without prior chemotherapy or radiotherapy (Table I). Written informed consent was obtained from each patient. Tissue samples were immediately stored in liquid nitrogen for further analysis.

Immunohistochemistry(IHC). TROP2 expression was detected by IHC in paraffin-embedded specimens, using the standard immunoperoxidase staining procedure. The $5 \mu \mathrm{m}$ thick slides were incubated overnight with anti-TROP2 antibody (1:50; Cell Signaling Technology, Inc., Danvers, MA, USA). Sections were then stained with Diaminobenzidine (DAB) and 
hematoxylin after the application of a secondary antibody for $30 \mathrm{~min}$ at room temperature. The degree of immunostaining was reviewed and scored independently by two observers as previously described $(6,28)$.

Cell culture. The normal osteoblast cells hFOB1.19 and OS cell lines U2OS, MG63, and MNNG/HOS were purchased commercially from Academia Sinica Cell Bank (Shanghai, China). HFOB1.19 cells were cultured in DMEM/F-12 (Thermo Fisher Scientific, Inc., Waltham, MA, USA) according to American Type Culture Collection (ATCC; Manassas, VA, USA) protocols. MNNG/HOS and MG63 cells were cultured in Eagle's minimum essential medium (Thermo Fisher Scientific, Inc.) containing 10\% FBS (15140-122; Gibco; Thermo Fisher Scientific, Inc.). The U2OS cells were cultured in Roswell Park Memorial Institute-1640 (RPMI-1640; Thermo Fisher Scientific, Inc.) medium containing 10\% FBS (15140-122; Gibco; Thermo Fisher Scientific, Inc.). Cells were cultured at $37^{\circ} \mathrm{C}$ in a humidified atmosphere of $5 \% \mathrm{CO}_{2}$.

Lentiviral and retroviral infection. A lentiviral short hairpin RNA (shRNA) construct targeting TROP2 was obtained from Jikai Corporation (Shanghai, China), with a sequence as follows: 5'-GCGGCAGAACACGTCTCAGAACTCGAGTTC TGAGACGTGTTCTGCCGC-3'. The oligonucleotides were phosphorylated, annealed, and cloned into the pLKO.1 vector according to the manufacturer's instructions. Lentiviral overexpression particles were prepared by GenePharma (Shanghai, China). Lentiviral and retroviral infection of MG63, U2OS and MNNG/HOS cells were performed according to the manufacturer's protocols. The expression of TROP2 was determined by western blot analyses and quantitative polymerase chain reaction (qPCR).

Cell proliferation. To evaluate the effects of TROP 2 on OS cells, MG63, U2OS and MNNG/HOS cells were seeded into 96-well plates on days 1, 2, 3, and 4 post-infection (3,000 cells/well). After $24 \mathrm{~h}$, the cell proliferation rate of MG63 and MNNG/HOS cells was detected with the CCK-8 Assay kit. Experiment was performed with three replicates.

Wound healing assay. For wound healing assay, MG63 and MNNG/HOS cells were seeded into six-well plates and cultured to $100 \%$ confluence. The cell layer was carefully scratched to create a wound using a sterile $1,000 \mu$ l pipette tip. Then, the cells were washed twice with PBS and treated with complete medium without FBS. The evaluation of wound healing was done under a light microscope at $24 \mathrm{~h}$ after scratching. The percentage of wound closure was calculated using Image J software (Rasband, W.S., Image J, U.S. National Institutes of Health, Bethesda, Maryland, USA).

Transwell assay. Transwell assay was performed to determine the effect of TROP2 on the migration of OS cells. Cells were suspended in serum-free medium and added to the upper chamber (Corning Costar, Rochester, NY, USA) and 10\% FBS-medium was added in the lower chamber. After incubation for $24 \mathrm{~h}$, the non-migratory cells were removed, while the migratory cells below the membrane were fixed and stained with crystal violet. The number of migration cells were counted under a light microscope (magnification, $\mathrm{x} 200$ ) using five randomly chosen visual fields.

$R N A$ isolation and reverse transcription $(R T)-q P C R$. Total cellular RNA was extracted from cell lines using Trizol reagent (Takara Bio, Inc., Otsu, Japan) according to the manufacturer's instructions. Total RNA was reverse-transcribed into cDNA by using the reverse transcriptional kit (Takara Bio, Inc.). The expression level of TROP2 was quantified by RT-qPCR using SYBR Green PCR PrimeScript RT-PCR Kit (Takara Bio, Inc.) on the ABI Step One Plus System. The following primers were used: TROP2, forward: 5'-CCTCATCGCCGTCATCGT-3' and reverse: 5'-CGGTTCCTTTCTCAACTCCC'; GAPDH, the internal control, were forward: 5'-GACTCATGACCACAG TCCATGC-3' and reverse: 5'-AGAGGCAGGGATGATGTT CTG-3'. The $2^{-\Delta \Delta C q}$ method was used to calculate relative gene expression.

Western blot analysis. Total protein of tissue samples and cells was extracted by using radioimmunoprecipitation assay buffer with a protease inhibitor (Beyotime Institute of Biotechnology, Haimen, China). After the protein concentration was measured by the BCA Protein Assay Kit (Beyotime Institute of Biotechnology), equivalent amounts of protein were separated by $10 \%$ sodium dodecyl sulfate-polyacrylamide gel and transferred onto a polyvinylidene fluoride membrane (EMD Millipore, Billerica, MA, USA). After blocking in 5\% nonfat milk for $1 \mathrm{~h}$, the polyvinylidene difluoride membranes were incubated overnight at $4^{\circ} \mathrm{C}$ with antibodies specific to TROP2 (1:1,000; Cell Signaling Technology, Inc.), $\beta$-actin $(1: 2,000$; Cell Signaling Technology, Inc.), p-phosphoinositide 3-kinase (p-PI3K; 1:1,000; Cell Signaling Technology, Inc.), PI3K (1:1,000; Cell Signaling Technology, Inc.), p-protein kinase B (p-AKT; 1:1,000; Cell Signaling Technology, Inc.), and AKT (1:1,000; Cell Signaling Technology, Inc.). Horseradish peroxidase-conjugated goat anti-rabbit secondary antibody (1:1,000; Beyotime Institute of Biotechnology) was applied for $2 \mathrm{~h}$ at room temperature. An enhanced chemiluminescent detection reagent (Bio-Rad Laboratories, Inc., Hercules, CA, USA) was used to detect immunoreactive bands, and all protein expression was quantified using Bio-Rad XRS chemiluminescence detection system (Bio-Rad Laboratories, Inc.).

Statistical analysis. All experiments were repeated at least three times. Results were presented as mean \pm standard deviation and analyzed using SPSS 23.0 software (IBM Corp., Armonk, NY, USA). Statistical differences between the means of the various groups were analyzed using one-way and two-way analysis of variance (for cell proliferation rate and TROP2 mRNA levels at all time points) followed by the post hoc Bonferroni's test. Differences between two groups were evaluated by using the Student's t-test when appropriate. For all analyses, $\mathrm{P}<0.05$ was considered to indicate a statistically significant difference.

\section{Results}

TROP2 was significantly up-regulated in OS cell lines and clinical specimens. RT-qPCR and western blotting analysis were employed to detect the expression of TROP2 in OS cell 
Table I. Clinical profiles of the 10 patients with osteosarcoma.

\begin{tabular}{lc}
\hline Clinical variable & No. of patients $(\mathrm{n}=10)$ \\
\hline Sex & 5 \\
Female & 5 \\
Male & \\
Age, years & 27.7 \\
Median & $9-64$ \\
Range & \\
Tumor location & 8 \\
Femur/tibia & 2 \\
Humerus & \\
Tumor size, cm & 4 \\
$\leq 5$ & 6 \\
$>5$ & \\
Distant metastasis & 0 \\
Yes & 10 \\
No & \\
\hline
\end{tabular}

lines. The results show that the expression of TROP2 was markedly up-regulated in three different OS cell lines (U2OS, MG63, and MNNG/HOS) compared to normal osteoblast cells hFOB1.19 ( $\mathrm{P}<0.05$; Fig. 1). To determine the expression of TROP2 in OS specimens and paired adjacent normal bone tissues, IHC was performed. Significantly higher TROP2 levels were identified in OS tissues than in adjacent normal bone tissues ( $\mathrm{P}<0.05$; Fig. 2$)$.

TROP2 promotes cell proliferation. The effects of TROP2 on the proliferation rate of OS cells were identified by CCK-8 assay, which revealed the overexpression of TROP2 significantly promoted the proliferation of MG63, U2OS and MNNG/HOS cells in a time-dependent manner. When TROP2 was knocked-down, the proliferation rate decreased significantly (Fig. 3, S1 file). In addition, to check the efficiency of TROP2 overexpression and knockdown, TROP2 mRNA levels at all time points after infection were detected and shown in S2 file.

TROP2 promotes cell migration. To explore the biological effects of TROP 2 on OS cell migration, a wound migration assay was performed. Relevant photographs were taken at 0 and $24 \mathrm{~h}$ after wound induction. Western blotting analysis showed successful upregulation and downregulation of TROP2 levels in OS cell lines (Fig. 4A and B). The wound-healing abilities of TROP2-overexpressing OS cells were significantly higher than those of the control group. In contrast, TROP2 depletion markedly decreased the wound-closure capacity (Fig. 4C and D). To further explore the function of TROP2 in OS, a Transwell migration assay was performed. As shown in Fig. 4E, migration was markedly increased in cells overexpressing TROP2. In contrast, TROP2 downregulation inhibited the migration of OS cells (Fig. 4E and F).

TROP2 knockdown inhibits the progression of OS by regulating the PI3K/AKT pathway. To explore the possible molecular mechanism underlying the effects of TROP 2 on OS cells, we detected the activation of PI3K/AKT signaling pathway, which is a key regulator of OS development. The result showed that TROP2 overexpression significantly upregulated the levels of p-PI3K and p-AKT. In addition, p-PI3K and p-AKT levels were decreased in TROP2-knockdown cells (Fig. 5A).

Blocking the PI3K/AKT signaling pathway rescues the increased cell proliferation and migration induced by TROP 2 overexpression. To verify the role of the PI3K/AKT signaling pathway, LY294002, a specific PI3K/AKT inhibitor, was used to inhibit p-AKT expression. The concentration of LY294002 used was $10 \mu \mathrm{M}$, based on previous studies (29-31). LY294002 significantly inhibited p-AKT expression (Fig. 5B). The migration ability of OS cells exposed to LY294002 were markedly decreased. The increased migration induced by TROP2 overexpression were also reduced by LY294002 exposure (Fig. 5C).

\section{Discussion}

Although outstanding advances in multiagent chemotherapy and surgical technique have been achieved in recent decades, the prognosis for OS patients with locally advanced or metastatic remains poor (2-4). Thus, there is an urgent need to research the molecular mechanisms and deregulated genes responsible for OS carcinogenesis. In this study, we found that TROP2 was significantly upregulated in human OS tissues. Overexpression of TROP2 increased the proliferation and migration of OS cells, while TROP2 knockdown significantly decreased cell growth and migration. Moreover, upregulation of TROP2 activated PI3K/AKT pathway. The increased migration observed after TROP2 overexpression were rescued by inhibition of PI3K/AKT. These novel findings indicate that TROP2 promotes OS cell proliferation and migration through PI3K/AKT signaling.

Previous studies have linked TROP2 to increased tumor growth and demonstrated TROP2 to be a candidate tumor prognostic marker in various tumors. TROP2 is found to be overexpressed in the majority of human epithelial cancers, including pancreatic cancer, gastric cancer, lung cancer, ovarian cancer, and colorectal cancers $(10,14,32)$. Chen et al (33) investigated the expression of TROP2 and epithelial-mesenchymal transition (EMT) indicator proteins in 93 patients with gallbladder cancer (GBC) and demonstrated that increased expression of TROP2 in GBC is associated significantly with aggressive progression and poor prognosis. In another study based on clinical samples, Guan et al (34) found that overexpression of TROP2 could be an independent predictor for poor clinical outcome in nasopharyngeal carcinoma. In cervical cancer cell line CaSki cells, Liu et al (35) found that Trop2 knockdown significantly inhibited the proliferation and colony formation of CaSki cells, and demonstrated that TROP2 might become a novel target for cervical cancer treatment. Similarly, in our study, IHC revealed higher TROP2 expression in OS samples, suggesting that high TROP2 expression may be associated with a poor prognosis. Overexpression of TROP2 increased the proliferation and migration of two different OS cell lines, indicating that high TROP2 levels may promote the generation, development, and metastasis of OS. 

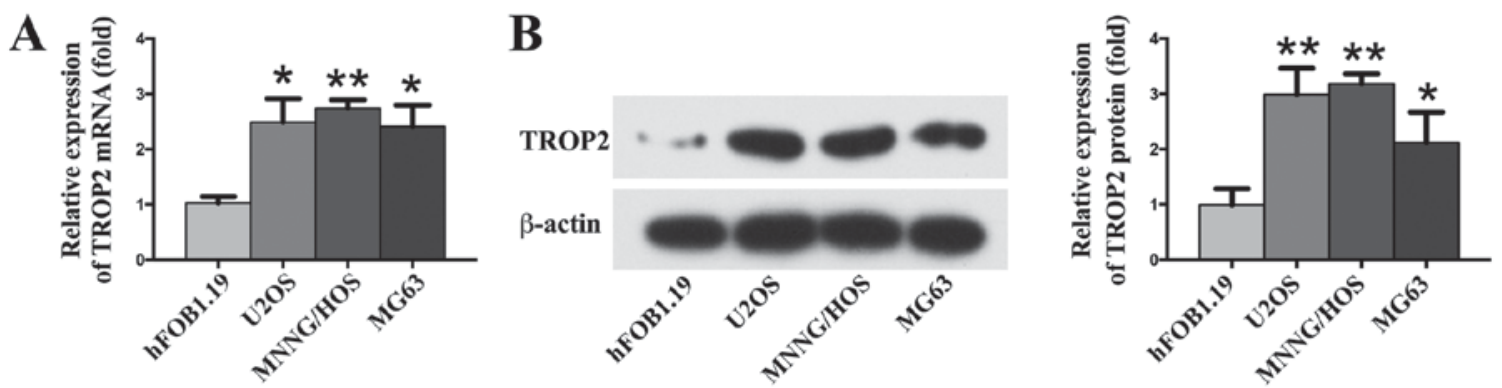

Figure 1. TROP2 is upregulated in OS cell lines. (A) Reverse transcription-quantitative polymerase chain reaction analysis revealed the expression levels of TROP2 in three OS cell lines (U2OS, MG63 and MNNG/HOS) and the normal osteoblast cells hFOB1.19. (B) Western blot analysis was performed to determine the expression of TROP2 in the OS cell lines (U2OS, MG63, and MNNG/HOS) and the normal osteoblast cells hFOB1.19. These results indicated that the expression of TROP2 was upregulated in OS cell lines. ${ }^{*} \mathrm{P}<0.05$ and ${ }^{* *} \mathrm{P}<0.01$ vs. hFOB1.19. TROP2, trophoblast cell surface antigen 2; OS, osteosarcoma.
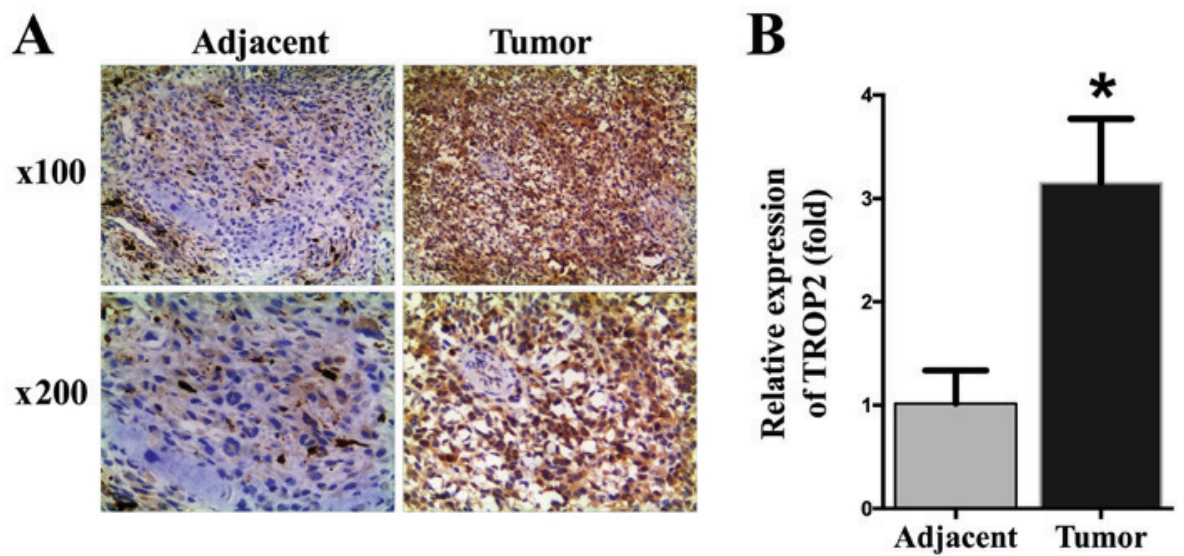

Figure 2. TROP2 is upregulated in clinical OS specimens. (A) Representative immunohistochemical staining image of OS tissues and paired adjacent normal bone tissues. Magnification, x100 and x200 as indicated. (B) Relative quantitative comparison of TROP2 expression in OS tissues and paired adjacent normal bone tissues. "P<0.05 vs. adjacent normal bone tissues. TROP2, trophoblast cell surface antigen 2; OS, osteosarcoma.
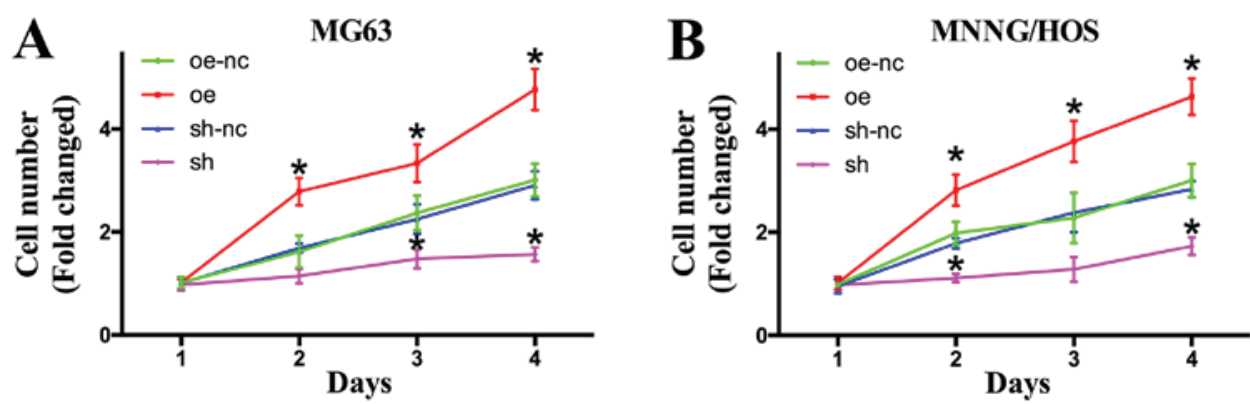

Figure 3. Effects of TROP2 overexpression and knockdown on OS cell proliferation. (A) Cell proliferation was assessed in MG63 cell lines by CCK-8 assay on days 1,2, 3 and 4 following lentiviral infection. (B) Cell proliferation was assessed in MNNG/HOS cell lines by CCK8 assay on days 1,2,3 and 4 following lentiviral infection. "P<0.05 vs. oe-nc. TROP2, trophoblast cell surface antigen 2; OS, osteosarcoma; Oe, overexpression of TROP2; sh, short hairpin RNA knockdown of TROP2; nc, negative control; oe-nc, the negative control group of TROP2 overexpression; sh-nc, the negative control group of TROP2 knockdown using nc short hairpin RNA; CCK-8, Cell Counting Kit-8.

The PI3K/AKT pathway plays a crucial role in tumorigenesis. Previous studies have shown that alteration of the $\mathrm{PI} 3 \mathrm{~K} / \mathrm{AKT}$ pathway is strongly implicated in OS pathogenesis. Moreover, studies have suggested that inhibition of the PI3K/AKT pathway disrupts functions essential for OS progression (36,37). Graziano et al (38) found that Wilms' tumor gene 1 (WT1) silencing inhibits OS cell proliferation by downregulating PI3K/AKT pathway. Huang and Jin (39) reported that the activation of the PI3K/AKT signal pathway is essential for the effects of zinc finger transcription factor ZIC2 on OS cells, and the effects of ZIC2 on the OS cells were reversed by a PI3K/AKT inhibitor. Interestingly, a previous study indicated that TROP2 promotes PI3K/AKT activation (40). Considering the critical role of PI3K/AKT in cell proliferation and migration, we assessed the levels of PI3K/AKT signaling pathway in OS cells. Being consistent with previous studies $(26,40,41)$, TROP2 increased the expression of total p-PI3K and p-AKT via PI3K/AKT pathway. Furthermore, the increased migration 
A

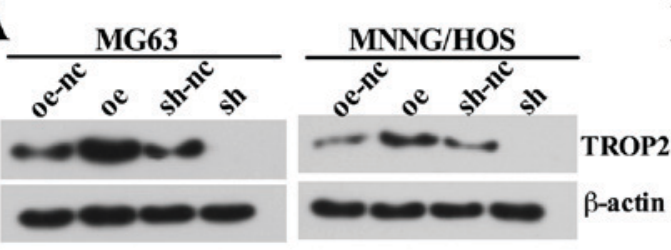

B

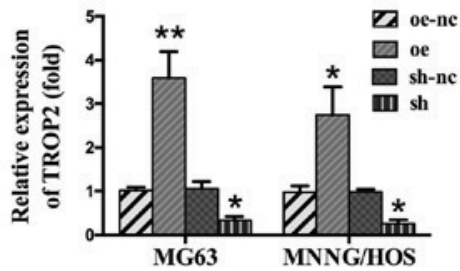

C
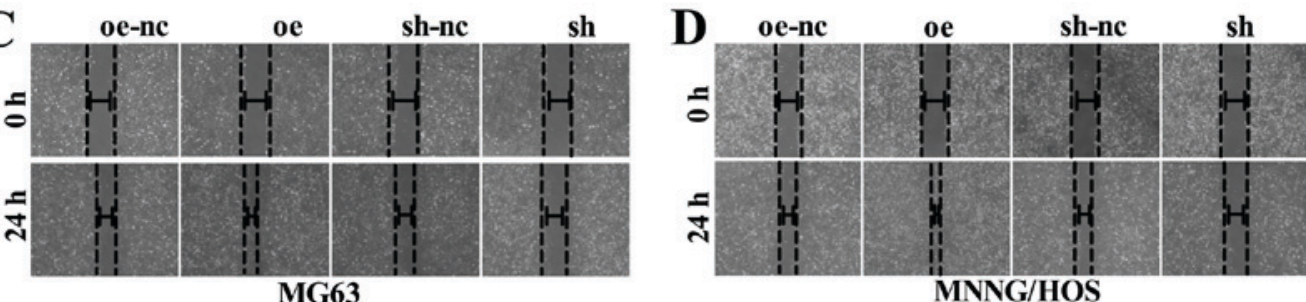

MNNG/HOS
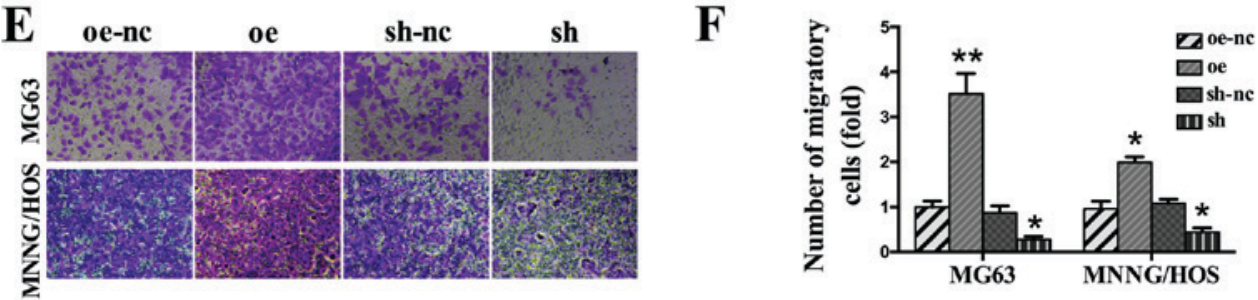

Figure 4. Effects of TROP2 knockdown and overexpression on the migration of OS cells. (A) Western blot analysis was performed to (B) confirm the upregulation of TROP2 protein expression by lentiviral infection, and the downregulation of TROP2 protein expression by shRNA in MG63 and MNNG/HOS cell lines. A wound-healing assay was performed to assess migration in the (C) MG63 and (D) MNNG/HOS cell lines (magnification, x100). (E) Transwell assays were performed to assess migration in the MG63 and MNNG/HOS cell lines (magnification, x200). (F) Relative quantitative comparison of migratory cells Images are representative of three independent experiments. All data are expressed as means \pm standard deviation. "P<0.05 and "** $\mathrm{P}<0.01$ vs. oe-nc. TROP2, trophoblast cell surface antigen 2; OS, osteosarcoma; Oe, overexpression of TROP2; sh, short hairpin RNA knockdown of TROP2; nc, negative control; oe-nc, the negative control group of TROP2 overexpression; sh-nc, the negative control group of TROP2 knockdown using nc short hairpin RNA.

A

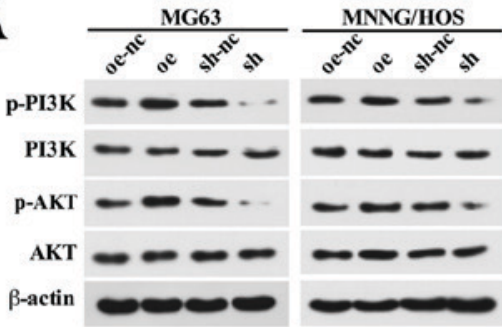

\section{B}

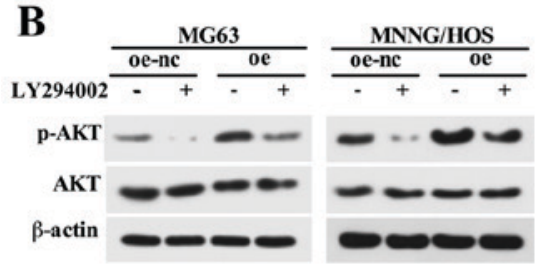

C

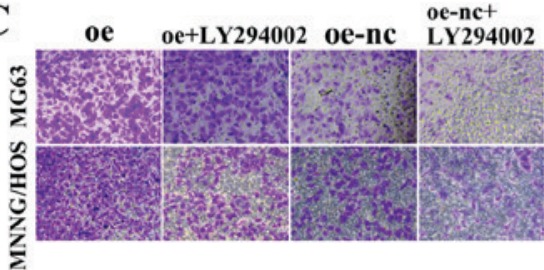

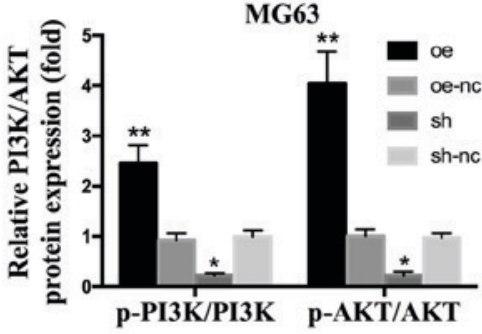
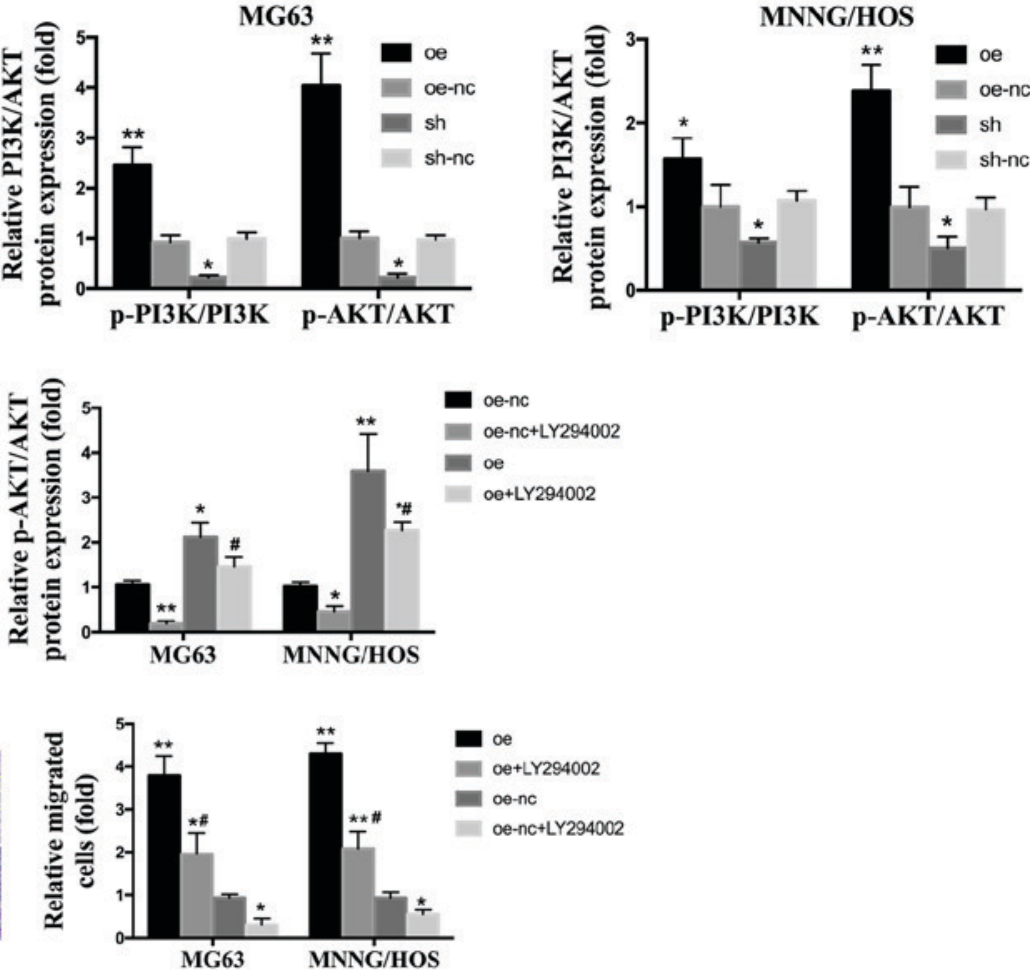

Figure 5. Effects of TROP2 knockdown and overexpression on the PI3K/AKT signaling pathway. (A) The protein levels of p-PI3K, PI3K, p-AKT and AKT were assessed by western blot analyses. (B) The effect of TROP2 overexpression on p-AKT expression level following the application of LY294002. (C) The effect of TROP2 overexpression on MG63 and MNNG/HOS cell migration following the application of LY294002 (magnification, $\mathrm{x} 200$ ). ${ }^{*} \mathrm{P}<0.05$ and ${ }^{* * *} \mathrm{P}<0.01$ vs. oe-nc; ${ }^{\prime} \mathrm{P}<0.05$ vs. oe group. TROP2, trophoblast cell surface antigen 2; OS, osteosarcoma; Oe, overexpression of TROP2; sh, short hairpin RNA knockdown of TROP2; nc, negative control; oe-nc, the negative control group of TROP2 overexpression; sh-nc, the negative control group of TROP2 knockdown using nc short hairpin RNA. 
and proliferation induced by TROP 2 overexpression was rescued by a PI3K/AKT inhibitor. Thus, we inferred that up-regulating TROP2 causes aberrant activation of PI3K/AKT in OS cells.

To the best of our knowledge, this is the first study of the effect of TROP2 on OS cells. However, some limitations of the present study should be noted. First, this was an in vitro study without in vivo evaluation, which may decrease the robustness of the results. Thus, further in vivo application of TROP2-related treatment, such as molecular targeted drug for TROP2 should be used to verify these findings. Second, as most of the findings in this study were obtained from MG63 and MNNG/HOS cells, more OS cell lines should be studied in the future. Third, due to the small patient population size, we have limited ability to reveal the correlation between TROP2 expression and the prognosis of OS. In addition, the carcinogenesis of OS is complicated, which means TROP2 may also target other signaling pathways, further studies are therefore required. Nevertheless, our study provides useful insight into the effect of TROP 2 on cell proliferation and migration in OS cell lines. Taken together, our data suggest that TROP2 promotes human OS cell proliferation and migration via activation of PI3K/AKT pathway.

\section{Acknowledgements}

Not applicable.

\section{Funding}

The present study received financial support from the Natural Science Foundation of China in Xinjiang Uygur Autonomous Region (grant no. 2014211C034).

\section{Availability of data and materials}

The datasets used and analyzed during the current study are available from the corresponding author on reasonable request.

\section{Authors' contributions}

ZT conceived and designed the present study. QG, AN and $\mathrm{XG}$ performed the experiments and were also the predominant contributors to the writing of the manuscript. KT and CL analyzed and interpreted the data. XF made contributions to the interpretation of data and critically revised the manuscript for important intellectual content. All authors read and approved the final manuscript.

\section{Ethics approval and consent to participate}

The present study was approved by the Medical Ethics Committee of The First Affiliated Hospital of Xinjiang Medical University (Xinjiang, China). Written informed consent was obtained from each patient.

\section{Consent for publication}

Not applicable.

\section{Competing interests}

The authors declare that they have no competing interests.

\section{References}

1. Zambo I and Vesely K: WHO classification of tumours of soft tissue and bone 2013: The main changes compared to the 3rd edition. Cesk Patol 50: 64-70, 2014 (In Czech).

2. Hu K, Wang Z, Lin P, Wen Z, Ren H, Sun L, Li H, Li B, Wang S, Zhou X, et al: Three hematological indexes that may serve as prognostic indicators in patients with primary, high-grade, appendicular osteosarcoma. Oncotarget 8: 43130-43139, 2017.

3. Li GL, Wu YX, Li YM and Li J: High expression of long non-coding RNA XIST in osteosarcoma is associated with cell proliferation and poor prognosis. Eur Rev Med Pharmacol Sci 21: 2829-2834, 2017.

4. Wu Y, Wu J, Dong QR and Guo NZ: Association between expression of nuclear receptor co-activator 5 protein and prognosis in postoperative patients with osteosarcoma. Oncol Lett 15: 1888-1892, 2018.

5. Tao Y, Xin M, Cheng H, Huang Z, Hu T, Zhang T and Wang J: TRIM37 promotes tumor cell proliferation and drug resistance in pediatric osteosarcoma. Oncol Lett 14: 6365-6372, 2017.

6. Lu J, Song G, Tang Q, Yin J, Zou C, Zhao Z, Xie X, Xu H, Huang G, Wang J, et al: MiR-26a inhibits stem cell-like phenotype and tumor growth of osteosarcoma by targeting Jagged1. Oncogene 36: 231-241, 2017.

7. Liu X, Zhou X, Xu H, He Z, Shi X and Wu S: SLC34A2 regulates the proliferation, migration, and invasion of human osteosarcoma cells through PTEN/PI3K/AKT signaling. DNA Cell Biol 36: 775-780, 2017

8. Cao J, Han X, Qi X, Jin X and Li X: TUG1 promotes osteosarcoma tumorigenesis by upregulating EZH2 expression via miR-144-3p. Int J Oncol 51: 1115-1123, 2017.

9. Zhao YX, Wang YS, Cai QQ, Wang JQ and Yao WT: Up-regulation of HDAC9 promotes cell proliferation through suppressing p53 transcription in osteosarcoma. Int J Clin Exp Med 8: 11818-11823, 2015.

10. Kong JS, Kim HJ, Kim MJ, Kim A, Lee D, Han K, Park S, Koh JS and Myung JK: The significance of TROP2 expression in predicting braf mutations in papillary thyroid carcinoma. J Pathol Transl Med 52: 14-20, 2018.

11. Zhao W, Zhu H, Zhang S, Yong H, Wang W, Zhou Y, Wang B, Wen J, Qiu Z, Ding G, et al: Trop2 is overexpressed in gastric cancer and predicts poor prognosis. Oncotarget 7: 6136-6145, 2016.

12. Shvartsur A and Bonavida B: Trop2 and its overexpression in cancers: Regulation and clinical/therapeutic implications. Genes Cancer 6: 84-105, 2015.

13. Lin H, Zhang H, Wang J, Lu M, Zheng F, Wang C, Tang X, $\mathrm{Xu}$ N, Chen R, Zhang D, et al: A novel human Fab antibody for Trop2 inhibits breast cancer growth in vitro and in vivo. Int J Cancer 134: 1239-1249, 2014

14. Mao Y, Wang X, Zheng F, Wang C, Tang Q, Tang X, Xu N, Zhang $\mathrm{H}$, Zhang $\mathrm{D}$, Xiong $\mathrm{L}$, et al: The tumor-inhibitory effectiveness of a novel anti-Trop2 Fab conjugate in pancreatic cancer. Oncotarget 7: 24810-24823, 2016.

15. Fong D, Moser P, Krammel C, Gostner JM, Margreiter R, Mitterer M, Gastl G and Spizzo G: High expression of TROP2 correlates with poor prognosis in pancreatic cancer. Br J Cancer 99: 1290-1295, 2008.

16. Mühlmann G, Spizzo G, Gostner J, Zitt M, Maier H, Moser P, Gastl G, Zitt M, Müller HM, Margreiter R, et al: TROP2 expression as prognostic marker for gastric carcinoma. J Clin Pathol 62: $152-158,2009$

17. Inamura K, Yokouchi $\mathrm{Y}$, Kobayashi M, Ninomiya $\mathrm{H}$, Sakakibara R, Subat S, Nagano H, Nomura K, Okumura S, Shibutani T and Ishikawa Y: Association of tumor TROP2 expression with prognosis varies among lung cancer subtypes. Oncotarget 8: 28725-28735, 2017.

18. Pak MG, Shin DH, Lee CH and Lee MK: Significance of EpCAM and TROP2 expression in non-small cell lung cancer. World J Surg Oncol 10: 53, 2012.

19. Wu B, Yu C, Zhou B, Huang T, Gao L, Liu T and Yang X: Overexpression of TROP2 promotes proliferation and invasion of ovarian cancer cells. Exp Ther Med 14: 1947-1952, 2017. 
20. Ohmachi T, Tanaka F, Mimori $\mathrm{K}$, Inoue $\mathrm{H}$, Yanaga $\mathrm{K}$ and Mori M: Clinical significance of TROP2 expression in colorectal cancer. Clin Cancer Res 12: 3057-3063, 2006.

21. SukhthankarM,AlbertiS andBaekSJ:(-)-Epigallocatechin-3-gallate (EGCG) post-transcriptionally and post-translationally suppresses the cell proliferative protein TROP2 in human colorectal cancer cells. Anticancer Res 30: 2497-2503, 2010.

22. Cubas R, Zhang S, Li M, Chen C and Yao Q: Trop2 expression contributes to tumor pathogenesis by activating the ERK MAPK pathway. Mol Cancer 9: 253, 2010.

23. Fang YJ, Lu ZH, Wang GQ, Pan ZZ, Zhou ZW, Yun JP, Zhang MF and Wan DS: Elevated expressions of MMP7, TROP2, and survivin are associated with survival, disease recurrence, and liver metastasis of colon cancer. Int J Colorectal Dis 24: 875-884, 2009.

24. Guan H, Guo Z, Liang W, Li H, Wei G, Xu L, Xiao H and Li Y: Trop2 enhances invasion of thyroid cancer by inducing MMP2 through ERK and JNK pathways. BMC Cancer 17: 486, 2017.

25. Chiang SF, Kan CY, Hsiao YC, Tang R, Hsieh LL, Chiang JM, Tsai WS, Yeh CY, Hsieh PS, Liang Y, et al: Bone marrow stromal antigen 2 is a novel plasma biomarker and prognosticator for colorectal carcinoma: A secretome-based verification study. Dis Markers 2015: 874054, 2015.

26. Yang J, Zhu Z, Wang H, Li F, Du X and Ma RZ: Trop2 regulates the proliferation and differentiation of murine compact-bone derived MSCs. Int J Oncol 43: 859-867, 2013.

27. Eisenwort G, Jurkin J, Yasmin N, Bauer T, Gesslbauer B and Strobl H: Identification of TROP2 (TACSTD2), an EpCAM-like molecule, as a specific marker for TGF- $\beta 1$-dependent human epidermal Langerhans cells. J Invest Dermatol 131: 2049-2057, 2011.

28. Wen ZQ, Li XG, Zhang YJ, Ling ZH and Lin XJ: Osteosarcoma cell-intrinsic colony stimulating factor- 1 receptor functions to promote tumor cell metastasis through JAG1 signaling. Am J Cancer Res 7: 801-815, 2017.

29. Gong C, Liao H, Wang J, Lin Y, Qi J, Qin L, Tian LQ and Guo FJ: LY294002 induces G0/G1 cell cycle arrest and apoptosis of cancer stem-like cells from human osteosarcoma via down-regulation of PI3K activity. Asian Pac J Cancer Prev 13: 3103-3107, 2012.

30. Han G, Wang Y and Bi W: C-Myc overexpression promotes osteosarcoma cell invasion via activation of MEK-ERK pathway. Oncol Res 20: 149-156, 2012.

31. Liu Y, Cheng Z, Pan F and Yan W: MicroRNA-373 promotes growth and cellular invasion in osteosarcoma cells by activation of the PI3K/AKT-Rac1-JNK pathway: The potential role in spinal osteosarcoma. Oncol Res 25: 989-999, 2017.
32. Son S, Shin S, Rao NV, Um W, Jeon J, Ko H, Deepagan VG Kwon S, Lee JY and Park JH: Anti-Trop2 antibody-conjugated bioreducible nanoparticles for targeted triple negative breast cancer therapy. Int J Biol Macromol 110: 406-415, 2018.

33. Chen MB, Wu HF, Zhan Y, Fu XL, Wang AK, Wang LS and Lei HM: Prognostic value of TROP2 expression in patients with gallbladder cancer. Tumour Biol 35: 11565-11569, 2014.

34. Guan GF, Zhang DJ, Wen LJ, Yu DJ, Zhao Y, Zhu L, Guo YY and Zheng Y: Prognostic value of TROP2 in human nasopharyngeal carcinoma. Int J Clin Exp Pathol 8: 10995-11004, 2015.

35. Liu X, Li S and Yi F: Trop2 gene: A novel target for cervical cancer treatment. J Cancer Res Clin Oncol 140: 1331-1341, 2014.

36. Ma J, Huang H, Han Z, Zhu C and Yue B: RLN2 Is a positive regulator of AKT-2-induced gene expression required for osteosarcoma cells invasion and chemoresistance. Biomed Res Int 2015: 147468, 2015.

37. Wang H, Luo QF, Peng AF, Long XH, Wang TF, Liu ZL, Zhang GM, Zhou RP, Gao S, Zhou Y and Chen WZ: Positive feedback regulation between Akt phosphorylation and fatty acid synthase expression in osteosarcoma. Int J Mol Med 33: 633-639, 2014.

38. Graziano AC, Cardile V, Avola R, Vicario N, Parenti C, Salvatorelli L, Magro G and Parenti R: Wilms' tumor gene 1 silencing inhibits proliferation of human osteosarcoma MG-63 cell line by cell cycle arrest and apoptosis activation. Oncotarget 8: 13917-13931, 2017.

39. Huang S and Jin A: ZIC2 promotes viability and invasion of human osteosarcoma cells by suppressing SHIP2 expression and activating PI3K/AKT pathways. J Cell Biochem 119: 2248-2257, 2018.

40. Li X, Teng S, Zhang Y, Zhang W, Zhang X, Xu K, Yao H, Yao J, Wang H, Liang $X$ and Hu Z: TROP2 promotes proliferation, migration and metastasis of gallbladder cancer cells by regulating PI3K/AKT pathway and inducing EMT. Oncotarget 8: 47052-47063, 2017.

41. Lin JC, Wu YY, Wu JY, Lin TC, Wu CT, Chang YL, Jou YS, Hong TM and Yang PC: TROP2 is epigenetically inactivated and modulates IGF-1R signalling in lung adenocarcinoma. EMBO Mol Med 4: 472-485, 2012. 